\title{
Rheumatic and oncological diseases - common challenges
}

\section{Krzysztof Jeziorski}

The Didactics Department, National Institute of Geriatrics, Rheumatology and Rehabilitation, Warsaw, Poland

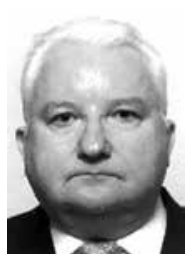

The progress of medicine leads to the creation of new medical specialties. Such is the case of internal medicine, from which, among others, rheumatology, geriatrics, cardiology and pulmonology developed. Dynamic progress of oncology and of its main specialties - oncological surgery, radiotherapy and clinical oncology (oncological chemotherapy) - led, on one hand to the emergence of the new specialties dedicated to specific organs (the process reflected by the establishment of specialized oncologic clinics) and on the other hand - to the emergence of new specialties dealing with the problems arising on the verge of already existing specialties e.g. cardio-oncology, neuro-oncology, dermato-oncology. The same observations apply to rheumatology, problems of which are a subject of interest to oncology.

Issues in which oncology and rheumatology overlap, include: 1) similar etiology of both types of diseases, 2) induction of rheumatic diseases by antineoplastic drugs and oncological diseases by antirheumatic drugs, and 3) paraneoplastic syndromes.

Rheumatic and neoplastic diseases have similar etiology. In their development genetic and environmental (viruses, chemical compounds, radiation) factors, as well as disorders of the immunological surveillance play a key role. It is known, that the antineoplastic drugs may induce the development of rheumatic diseases. The therapy with bleomycin, vinblastine, vincristine or cisplatin can result in the development of Raynaud's syndrome. The reactive arthritis as a result of BCG vaccine use in the bladder cancer therapy, as well as lupus-like syndrome after the interferon $\alpha$ (IFN- $\alpha$ ) and IFN- $\gamma$ therapy have been observed. The introduction of growth factors (G-CSF and GM-CSF) to oncology, used in conjunction with chemotherapy, caused patients to display acute symmetric inflammatory arthropathy. The term post-chemotherapy rheumatism has been accepted in the literature, such a condition occurring after cytostatic therapy with cisplatin, 5-fluorouracil, cyclophospha- mide, and methotrexate (most frequently in the therapy of mammary and ovarian cancer and non-Hodgkin lymphoma), hormonotherapy (tamoxifen) or interleukin 2 (IL-2) therapy. What is interesting, cyclophosphamide and methotrexate are also used in the treatment of rheumatic diseases. The symptoms of rheumatic disease can appear in few weeks' or months' time from the end of the chemotherapy; the premature menopause and disorders of the immune system are the mechanisms through which these symptoms develop. As it has been mentioned above, drugs used in the therapy of rheumatic diseases can lead to the development of the neoplasm, with cyclophosphamide causing the development of the bladder and skin cancer, as well as of lymphoproliferative disorders [1]. As other drugs finding use in rheumatology and at the same time contributing to the development of the neoplastic diseases, methotrexate, azathioprine and tumor necrosis factor inhibitors must be mentioned [2]. Paraneoplastic syndromes can be defined as symptoms concurrent to the neoplastic disorder, which can imitate other diseases and manifest themselves with various intensity. It is distinctive, that paraneoplastic syndromes are associated with most common neoplastic diseases - pulmonary cancer (adenocarcinoma in particular), pulmonary metastases, mammary, ovarian, colorectal and gastric cancers, non-Hodgkin lymphomas. The etiology of many of these syndromes is unknown, but most often they are caused by the excessive secretion of various substances - both those present physiologically and those found only in specific pathological circumstances - by the neoplasm. Mechanisms underlying the development of the paraneoplastic syndrome, include: secretion of hormones, growth factors, cytokines, and cytotoxic factors by the neoplasm itself or by the organism in reaction to the neoplasm presence, autoimmunization resulting from the interaction of circulating neoplastic antibodies with normal tissues, hyperreactivity to neoplastic antigens 
(which can subsequently result in a tissue destruction), abnormal immunologic reactions effecting from the cross-reaction between the neoplastic and normal tissue antigens. The production of substances causing the paraneoplastic syndromes depends on the embryogenesis of the tissues, from which the neoplasm originated. We recognize i.a. secretive (humoral), neuro-muscular, dermal, hematological syndromes, as well as those linked to the symptoms associated with the digestive tract, urinary tract, circulatory system and connective tissue disorders. The latter ones are subject of interest of both rheumatology and oncology. They constitute a significant diagnostic and therapeutic problem, as the occurring specific and unspecific symptoms, can suggest the presence of neoplastic disease, as well as of rheumatic one. Such unspecific symptoms, as: fever, fatigue, body mass loss or loss of appetite are typical both for rheumatic and proliferative (Hodgkin's and non-Hodgkin lymphoma, myeloid leukemia) diseases. In turn, the symptoms of paraneoplastic syndromes can be characteristic and point to the rheumatic disease, such as i.a.: undifferentiated arthritis, dermatomyositis, lupus-like syndrome, Raynaud's syndrome, fasciitis, erythema nodosum, and complex regional pain syndrome. A characteristic feature of the paraneoplastic diseases is the withdrawal of their symptoms in the course of the anti-neoplastic therapy and their intensification at the time of the progression or relapse of the neoplasm. This enables some level of differentiation between paraneoplastic syndromes and rheumatic diseases. The issue of selecting rheumatic patients who should be diagnosed for neoplastic diseases remains still unresolved.

It is accepted, that, from the clinical and economical point of view, the diagnosis of the rheumatic patients for the presence of neoplastic diseases is not recommended, apart from in cases of: 1) documented history of the neoplastic disease, including family history, 2) previous exposition to carcinogens, 3) atypical course of the disease, 4) asymmetric or sudden arthritis occurring at old age, 5) presence of symptoms not proportional to the level of inflammation, 6) occurrence of paraneoplastic syndromes, 7) the failure of the standard therapeutic treatment, and 8) elevated levels of neoplastic markers [3-5].

In conclusion: differentiation between paraneoplastic syndromes and rheumatic diseases in some situations often requires specialist knowledge and consultations. Certain clinical situations described above require the exclusion of cancer before starting treatment for rheumatic disease.

The author declares no conflict of interest.

\section{References}

1. Kinlen LJ. Incidence of cancer in rheumatoid arthritis and other disorders after immunosuppressive treatment. Am J Med 1985; 21: 44-49.

2. Beauparlent P, Papp K, Haraoui B. The incidence of cancer associated with treatment of rheumatoid arthritis. Semin Arthritis Rheum 1999; 29: 148-154.

3. Alias A, Rodrigez EJ, Bateman HE, et al. Rheumatology and oncology. An updated review of rheumatic manifestations of malignancy and anti-neoplastic therapy. Bulletin of the NYU Hospital for Joint Diseases 2012; 70: 109-114.

4. Racanelli V, Prete $M$, Minoia $C$, et al. Rheumatic disorders as paraneoplastic syndromes. Autoimmun Rev 2008; 7: 352-358

5. Fam AG. Paraneoplastic rheumatic syndromes. Baillieres Best Pract Res Clin Rheumatol 2000; 14: 515-533. 